Methods Patients undergoing Y-stent assisted coil embolization in the ATLAS IDE trial (Investigational Device Exemption) were identified. The primary efficacy end point was complete aneurysm occlusion (Raymond-Roy class 1) on 12-month angiography, in the absence of retreatment or parent artery stenosis $(>50 \%)$ at the target location. The primary safety end point was any major stroke or ipsilateral stroke or neurological death within 12 months. Adjudication of the primary end points was performed by an independent Imaging Core Laboratory and the Clinical Events Committee.

Result A total of 60 patients of were identified. The mean age was 59 and $28.3 \%$ were men. The median aneurysm size was $6.7 \mathrm{~mm}$ with a median neck size of $4.3 \mathrm{~mm}$. Aneurysm locations were as follows: basilar apex (56.7\%), basilar trunk (3.3\%), anterior communicating artery (20\%), anterior cerebral artery $(3.3 \%)$, internal cerebral artery $(3.3 \%)$ and middle cerebral artery (13.3\%). The composite primary efficacy end point of complete aneurysm occlusion (Raymond-Roy 1) without parent artery stenosis or aneurysm retreatment was achieved in $81.1 \%$ of patients. Overall, $1.7 \%(1 / 60)$ of patients experienced a primary safety end point of major ipsilateral stroke or neurological death.

Conclusions In the ATLAS IDE aneurysm cohort premarket approval study, the Neuroform Atlas stent with adjunctive Ystent coiling met the primary end points and demonstrated high rates of long-term complete aneurysm occlusion at 12 months, with low rates of morbidity.

Disclosures A. Jadhav: None. S. Desai: None. R. Hanel: None. D. Frei: None. A. Khaldi: None. S. Hetts: None. O. Zaidat: None. B. Jankowitz: None.

\section{E-051 DISPARITIES IN STROKE: ASSOCIATING SOCIOECONOMIC FACTORS WITH ISCHEMIC STROKE OUTCOME}

A Nguyen*, GM Cortez, M Baretta, A Aghaebrahim, E Sauvageau, R Hanel. Neurosurgery, Baptist Health System- Jacksonville, Jacksonville, FL

10.1136/neurintsurg-2021-SNIS. 146

Introduction Socioeconomic status (SES) is a dynamic determinant factor that could predict the population's wellness in many aspects. However, there is little existing literature on how socioeconomic conditions influence ischemic stroke outcomes. This study aims to determine whether SES has any correlation with the functional outcome of ischemic stroke patients undergoing mechanical thrombectomy.

Methods Data was retrospectively collected using the Lyerly neurosurgery center's prospectively maintained thrombectomy database. Patient-level data collected include stroke risk factors such as age, race, gender, comorbidities, infarct core volume, history of stroke, and tobacco usage. We measured the outcome of ischemic stroke using the modified Rankin Score (mRS) at 90-day post-procedure. SES was defined by a combination of the zip code median income data we collected from the 2019 U.S. Census Bureau's American Community Survey (ACS) 5-year Projection and patients' health insurance coverage. We used several multivariate analyses to plot the correlation between the outcomes and SES.

Results Among 508 patients collected, 234 (46\%) had preferred outcome (mRS $\leq 2)$ and $274(54 \%)$ had poor outcome $(\mathrm{mRS}>2)$. Baseline risk factors that presented a significant correlation with poor outcome included age $(\mathrm{p}<0.001)$, infarct core volume $(p=0.007)$, hypertension $(p=0.006)$, atrial fibrillation $(\mathrm{p}=0.001)$, and existing history of stroke $(\mathrm{p}=0.012)$. After adjusting for nuisance variables, unfavorable functional outcome $(\mathrm{p}<0.001)$ was more likely to occur in patients with lower median income based on zip code. There was no association between health insurance and functional outcome $(\mathrm{p}=0.58)$.

Conclusions Median income based on zip code is a SES indicator that is potentially associated with functional outcomes of ischemic stroke patients undergoing mechanical thrombectomy. Further studies addressing this relationship are needed.

Disclosures A. Nguyen: None. G. M. Cortez: None. M. Baretta: None. A. Aghaebrahim: None. E. Sauvageau: None. R. Hanel: None.

\section{E-052 PREDICTORS OF PROLONGED CLOT ACCESS IN ACUTE ISCHEMIC STROKE: IMPLICATION OF VESSEL MORPHOLOGY}

GM Cortez* , A Monteiro, A Nguyen, A Aghaebrahim, E Sauvageau, R Hanel. Neurosurgery, Baptist Health System- Jacksonville, Jacksonville, FL

\subsection{6/neurintsurg-2021-SNIS. 147}

Background Therapeutic workflow processes have been implemented and improved over the years to enable rapid response for acute ischemic stroke patients. Optimization of mechanical thrombectomy devices and techniques overcame some of the initial technical constraints; nonetheless, the implication of vessel tortuosity in procedure time and outcomes is still uncertain. We aimed to evaluate the predictors and the impact of difficult catheter access to the target occluded vessel.

Methods This is a retrospective, single-center study. Baseline characteristics were retrieved, and adjudication of the aortic arch and cervical vasculature tortuosity was performed. The primary outcome of interest was to evaluate the factors associated with an increased puncture to clot time. Secondary outcomes included first pass, successful recanalization, and good clinical outcome.

Results A total of 183 patients met inclusion criteria (mean age $72.0 \pm 14.5,54.1 \%$ were women). Fifty-two patients (28.4\%) had an aortic arch type 1, 58 (31.7\%) type 2, and $73(39.9 \%)$ type 3 . Tortuosity was considered significant in 68 (37.1\%), and 103 (57.9\%) of brachiocephalic/common carotids and internal carotid arteries, respectively, and 38 (20.8\%) patients had a bovine aortic arch. The mean time from puncture-to-clot access was $15.8 \pm 8.7 \mathrm{~min}$, and punctureto-recanalization was $34.5 \pm 18.5$. Age, hyperlipidemia, aortic arch type III, ICA tortuosity, BC/CCA tortuosity, and BAD score demonstrated a positive correlation $(p<0.05)$ with increased time from puncture-to-clot access. The likelihood of achieving FPE was inversely correlated to the presence of tortuosity in the ICA $(p=0.02)$. None of the investigated anatomical factors demonstrated a correlation with functional outcomes.

Conclusion Unfavorable vascular anatomy is associated with prolonged clot access in patients undergoing endovascular thrombectomy. The factors associated with prolonged access time included age, hyperlipidemia, type III aortic arch, and the presence of tortuosity at the cervical vasculature (BC/CCA and ICA). Non-invasive imaging evaluation may allow prompt selection of the best approach and culminate in procedure optimization. 
Disclosures G. M. Cortez: None. A. Monteiro: None. A. Nguyen: None. A. Aghaebrahim: None. E. Sauvageau: None. R. Hanel: None.

\section{E-053 MOUNT EVEREST TECHNIQUE (MET) WITH THE NOVEL TENZING INSERTS AND SUPER LARGE BORE CATHETERS - TECHNIQUE AND INITIAL EXPERIENCE}

F Massari, J Singh, A Kuhn, V Naragum, V Anagnostakou, M Gounis, A Puri. Department of Radiology, University of Massachusetts Medical School, Worcester, MA

\subsection{6/neurintsurg-2021-SNIS.148}

Purpose To report the feasibility, safety and efficacy of the large bore catheters with matching Tenzing inserts for first pass efficacy in stroke thrombectomy without need for crossing the clot.

Methods We performed a retrospective review of our prospectively maintained neurointerventional database of mechanical thrombectomy for stroke. Patient demographics, clinical information, procedural and radiographic data was collected.

Results The most widely used techniques for stroke thrombectomy are aspiration, stent-retrievers or combination of these. The Mount Everest Technique (MET) has been designed combining innovative technology and the "Law of Conservation of Matter", taking advantage of not needing to cross the clot with the bigger catheters to "ingest" the clot with very high first pass success. The Mount Everest Technique (MET) entails a triaxial assembly with an 8 Fr 0.106 " Guiding Catheter (Base Camp), a 088" HiPoint "catheter on a stick/wire" with matching self-centering Tenzing insert catheter. The Tenzing catheter is used to climb up to the clot then, while pulling this insert out, the 088 " catheter is capable to progress into the clot "ingesting" it, thanks to the "Law of Conservation of Matter". The aspiration force is applied from the Base Camp catheter with the 088" HiPoint within the clot as a single assembly, thus taking the advantage of the super large bore distal catheter and the stable 0.106" Base Camp, resulting in the capability of physically "ingest" the clot. At the time of the abstract submission, we have utilized this novel Mount Everest Technique (MET) in 6 patients with large vessel occlusions involving the anterior circulation (ICA T-terminus and MCA M1 occlusions), with single pass (5) and 2 passes (1) complete vascular reperfusion of the occluded vessel in all the cases (TICI 3). No embolization in previously unaffected vascular territories or post procedure $\mathrm{SAH}$ has been observed.

Conclusion The Mount Everest Technique (MET) combines the technological novelty of the 0.106" Base Camp, 088" HiPoint and matching self-centering Tenzing insert catheters to navigate these super large bore catheters to the face of the clot without needing to cross them with large microcatheters or wires. Thereafter based on the "Law of Conservation of Matter", the retraction of the Tenzing insert while applying a suction force at the HiPoint catheter results in the "ingestion" of the clot in toto. This technique and catheter combination have led to $100 \%$ TICI 3 recanalization with $80 \%$ first pass success in our initial experience.

Disclosures F. Massari: None. J. Singh: None. A. Kuhn: None. V. Naragum: None. V. Anagnostakou: None. M. Gounis: None. A. Puri: None.
THE RIVER TRIAL: A PROSPECTIVE SINGLE ARM TRIAL OF STENTING THE TRANSVERSE SIGMOID SINUSES WITH THE RIVER STENT IN PATIENTS WITH IDIOPATHIC INTRACRANIAL HYPERTENSION RESISTANT TO MEDICAL THERAPY

A Patsalides*. Northwell Health, Manhasset, NY

10.1136/neurintsurg-2021-SNIS. 149

Background Venous sinus stenting (VSS) is increasingly used in the management of Idiopathic Intracranial Hypertension (IIH), using off label commercially available stents designed for arterial stenting. The River ${ }^{\mathrm{TM}}$ stent is a stent designed for VSS: it is a long $(7 \mathrm{~cm})$ open cell stent available in 3 diameters (6 to $9 \mathrm{~mm}$ ), with the flexibility to easily negotiate the tortuosity of the sigmoid sinus. The River trial is an HDE pivotal trial negotiated with the FDA to show safety and probable efficacy of the River ${ }^{\mathrm{TM}}$ stent in patients with moderate and severe IIH resistant to medical therapy.

Methods The River trial is a prospective, multicenter, single arm, open label clinical trial.Population: Patients with IIH (modified Dandy criteria), cerebrospinal fluid opening pressure $>25$, bilateral transverse-sigmoid sinus stenosis or unilateral stenosis of a dominant sinus with pressure gradient across the lesion $\geq 8 \mathrm{mmHg}$, and moderate to severe symptoms resistant to medical therapy. Patients had to have severe headaches (HIT-6 $\geq 59$ ) or moderate to severe visual field loss (MD between -6 and -30 ). A total of 39 subjects will be enrolled. Primary safety endpoint is the absence of major adverse events (MAE) at 12 months. MAE include stroke, neurological death, thrombosis or perforation of the sinus or cerebral vein and need for target vessel recanalization or IIH alternate surgical procedure.Primary benefit endpoint is the composite at 12 months of the absence of significant stenosis of the stented sinus and clinically relevant symptomatic improvement.

Results As of March 2021, 25 subjects have been enrolled. The River ${ }^{\mathrm{TM}}$ stent tracked easily and all procedures went smoothly with no complications. 19 subjects completed the 3-month follow-up, 18 completed the 6-month follow-up, and 16 subjects completed the 1-year follow-up visit. In addition, 3 subjects have completed the 2-year follow-up visit. Two subjects discontinued from the study after treatment with the River stent: one was lost to follow-up and one had a shunt placement prior to completing the 1-year visit. There were 39 adverse events reported in 17 subjects: 3 events in 3 subjects were considered serious, none were related to the study device or procedure; there were no unanticipated adverse device effects (UADEs); no deaths or stroke have occurred.Two subjects had symptomatic restenosis at the one-year follow-up and were treated with additional stenting of the superior sagittal sinus (two) and gastric by-pass (one). One patient had restenosis without recurrent symptoms and did not require additional treatment. One subject with persistent headaches at 6 months was shunted. The other subjects are doing well with significant improvement of headaches and or visual field.

Conclusions The results so far have shown safety and probable efficacy. Enrollment and follow-up are ongoing and updated results will be presented at the conference.

Disclosures A. Patsalides: None. 